J. Lake Sci. (湖泊科学), 2012, 24(4): 562-570

http: //www.jlakes.org. E-mail : jlakes@niglas.ac.cn

(C) 2012 by Journal of Lake Sciences

\title{
淹水环境中芦苇幼苗对两种底质的生理响应及其恢复状况”
}

\author{
柏 祥 $^{1,2}$, 陈开宁 ${ }^{1 * *}$, 任奎晓 ${ }^{1}$, 黄 蔚 $^{1}$, 陈效民 ${ }^{2}$,杨 华 ${ }^{3}$ \\ ( 1 : 中国科学院南京地理与湖泊研究所湖泊与环境国家重点实验室,南京 210008) \\ (2:南京农业大学资源与环境科学学院,南京 210095) \\ ( 3 : 巢湖市环境监察支队,巢湖 238000 )
}

\begin{abstract}
摘 要: 探讨湿地植物对淹水的响应及其恢复生长状况对于湖泊湿地的修复具有重要意义. 以芦苇 (Phragmites communis) 为对象, 研究其在湖泊沉积物 (LS) 和农田土壤 (AS) 两种底质上完全淹水一个月内每隔 $5 \mathrm{~d}$ 其叶片相对叶绿素含量 (rChlc) 、丙二醛含量 (MDA) 和叶绿素苂光特性的变化,并分别于淹水后的 $10 、 20$ 和 $30 \mathrm{~d}$ 对其恢复状况进行研究. 结果表 明:缓苗期间生长于 LS 上芦苇的 rChlc 和叶绿素苂光特性均高于生长于 AS 上的, 淹水后 $\mathrm{rChlc}$ 逐渐下降, $20 \mathrm{~d}$ 后下降趋 势更加明显, $30 \mathrm{~d}$ 时 LS 和 AS 上芦苇叶片的 rChlc 分别下降了 $40.82 \%$ 和 $39.49 \%$, MDA 则逐渐升高, 且生长于 AS 上的要 高于 LS 上的; 叶绿素苂光参数 $F v / F m$ 和 $Y$ 也均逐渐下降, 且总体上与 rChlc 的变化呈显著正相关; LS 上芦苇叶片的快速 光响应曲线 (RLCs) 在淹水后缓慢下降, 至 $25 \mathrm{~d}$ 后下降明显, 而 AS 上的则在 $15 \mathrm{~d}$ 后即显著下降. 淹水胁迫解除后, 两种底 质上芦苇的 rChlc 和叶绿素荧光特性均逐渐升高, 总体上 AS 上芦苇的增幅大于 LS 上的,但 LS 上的各指标仍大于 AS 上 的, 而 MDA 逐渐下降, 同样 AS 上的降幅要大于 LS 上的; 随着淹水时间的延长, 芦苇恢复到正常生长状态的时间有所增 加, 淹水 $30 \mathrm{~d}$ 的芦苇叶片调落并萌发出新叶和新的植株, 同时 LS 上芦苇的恢复时间要短于 AS 上的. 可见, 淹水抑制了幼 苗期芦苇的生长, 且随着时间的延长其抑制程度逐渐加重; 淹水时间短时芦苇能较快地恢复生长, 淹水时间长时则需要 长出新叶和萌发出新的植株来适应生长, 同时营养状况良好的底质在增加芦苇耐淹性及加快其淹水后的恢复生长方面 具有一定的积极意义.
\end{abstract}

关键词: 淹水;芦苇;叶绿素苂光;恢复状况;巢湖;底质

\section{Physiological response of Phragmites communis seedling to two types of sediment under submergence and its recovering growth}

\author{
BAI Xiang ${ }^{1,2}$, CHEN Kaining ${ }^{1}$, REN Kuixiao ${ }^{1}$, HUANG Wei ${ }^{1}$, CHEN Xiaomin ${ }^{2}$ \& YANG Hua ${ }^{3}$ \\ (1: State Key Laboratory of Lake Science and Environment, Nanjing Institute of Geography and Limnology, Chinese Academy \\ of Sciences, Nanjing 210008, P. R. China) \\ (2: College of Resources and Environmental Science, Nanjing Agricultural University, Nanjing 210095, P. R. China) \\ (3: Environment Monitoring Detachment of Chaohu City, Chaohu 238000, P. R. China)
}

\begin{abstract}
It's important to study the response of wetland plants to submergence and its recovery growth status for lacustrine wetland restoration. In this study, we revealed the varieties of relative chlorophyll content ( $\mathrm{rChlc}$ ), malondi-aldehyde content ( MDA) and chlorophyll fluorescence characteristics of Phragmites communis in submergence every $5 \mathrm{~d}$ during one month in two types of sediment (one from Lake Chaohu (LS), and the other from farmland (AS)), and its recovery status in $10 \mathrm{~d}, 20 \mathrm{~d}$ and $30 \mathrm{~d}$ after submergence. Results showed that, compared to AS, P. communis growing in LS had higher rChlc and chlorophyll fluorescence characteristics during the rejuvenate period. rChlc decreased after submergence and obviously after $20 \mathrm{~d}$, and further decreased by $40.82 \%$ and $39.49 \%$ in $30 \mathrm{~d}$ in LS and AS, respectively. However, MDA increased, and it was higher in AS than in LS. Chlorophyll fluorescence parameters $F v / F m$ and $Y$ both decreased, with significant correlation with rChlc varieties as a whole. The rap-

* 国家水体污染控制与治理科技重大专项项目(2008ZX07103-005,2011ZX07103-003) 和江苏省科技计划社会发展项 目 (BE2009603) 联合资助. 2011-08-22 收稿;2011-10-18 收修改稿. 柏祥, 男, 1984 年生, 博士研究生; E-mail: baix04@163.com.

** 通信作者; E-mail :knchen@ niglas. cn.
\end{abstract}


id light curves (RLCs) of $P$. communis growing in LS decreased slowly in $25 \mathrm{~d}$, comparing to an obvious decrease in $15 \mathrm{~d}$ in AS. After submergence, rChlc and chlorophyll fluorescence characteristics of $P$. communis in two sediments all increased, with a greater range of the plant in AS than that in LS; however, they were both higher in LS than those in AS, and MDA had a same decreasing trend. It took longer time for $P$. communis to recover to the normal growth status with the extension of submergence, the leaves withered after $30 \mathrm{~d}$ submergence and sprouted new leaves and plant. At the same time, the recovery time was shorter of the plant grown in LS than that in AS. Consequently, submergence inhibits the growth of $P$. communis, and the inhibited effect is greater with the extension of submergence. It takes a short time for plant to recover to the normal growth status when in short submergence, and it needs to sprout new leaves and plants to adapt when in long submergence. Simultaneously, it's meaningful for P. communis to increase its submergence tolerance and recover growth after submergence when growing in sediment with relative better nutritional status.

Keywords: Submergence; Phragmites communis; chlorophyll fluorescence; recovery status; Lake Chaohu; sediment

水位波动作为湿地重要的水文情势, 是控制湿地特征的关键因子,也是湿地典型的扰动特征之一,直接 影响湿地生态系统中植物群落的分布、生产量、稳定性、演替和物种多样性等 ${ }^{[-2]}$. 由于湿地水位的波动, 湿 地中的一些植物在其生活史中通常要经历一段或周期性的水淹 ${ }^{[3]}$, 影响这些植物个体的生长发育等. 底质 是湿地植物固着的基础和植物生长所需矿物质的主要来源 ${ }^{[4]}$, 在一定程度上影响湿地植物的个体生长和种 群结构. 湿地植物以其自身独有的特性成为湿地生态系统中的重要组成部分 ${ }^{[5]}$, 在为次级生产者和消费者 提供物质和能量的同时也为其它水生动物等提供了庇护和繁殖场所. 另外, 湿地植物的生长能够吸收水体 和沉积物中的污染物质, 达到净化水质的目的, 同时湿地植物的存在能够为微生物的生存和生长提供适宜 的环境, 因此利用湿地植物净化水质在富营养化水体修复中得到了广泛应用 ${ }^{[6]}$. 芦苇 (Phragmites communis) 隶属于禾本科芦苇属, 以其适应性广、抗逆性强等特点被广泛应用于湖泊、河流等湿地的恢复中.

巢湖位于安徽省中部, 属于长江下游的左岸水系,巢湖闸的修建使巢湖的水量发生了很大变化, 原来的 枯水季节也可出现高水位, 而丰水季节也可出现低水位, 这直接导致巢湖水生植物的覆盖度由 $30 \%$ 下降到 $1 \%$ 左右, 因此水位的变化可能是巢湖水生植物衰退的根本原因 ${ }^{[7]}$. 据监测资料显示, 巢湖的水位变幅可达 $3.5 \mathrm{~m}$, 且高水位持续时间可达 1 个月之久. 另外, 自 1970s 以来, 随着巢湖周边地区工农业的发展, 污水排 放、化肥和农药的大量施用等使大量点源及非点源等外源污染物输人, 导致巢湖水质急剧下降, 蓝藻水华频 繁暴发 ${ }^{[8-9]}$, 这在一定程度上也加速了巢湖水生植物的消失. 因此在巢湖湖岸带进行湿地恢复对于巢湖富营 养化的治理具有重要作用. 鉴于湿地恢复的迫切性及巢湖水位波动大的特点, 本文以芦苇为对象, 以叶绿素 苂光技术为主要手段研究了淹水环境中在两种不同底质上其幼苗生长的响应差异, 并对不同淹水时间后的 植物恢复状况一并进行了研究, 以期为巢湖湖滨带的湿地恢复提供科学参考.

\section{1 材料与方法}

\section{1 实验材料}

2010 年 8 月初在巢湖湖滨自然湿地中选取长势良好、生长一致的芦苇幼苗 (株高 $20 \mathrm{~cm}$ 左右)连根挖出 后带回湖边的实验基地, 栽植于高 $35 \mathrm{~cm}$ 、上口直径 $20 \mathrm{~cm}$ 的塑料桶中, 每桶栽种 2 株. 桶中底质分别为湖泊 沉积物 ( LS, 灰色粉沙淤泥) 和湖边农田土壤 (AS, 黄色粘土), 底质深度为 $30 \mathrm{~cm}$. 两种底质的基本特征见表 $1, \mathrm{LS}$ 的营养盐含量明显高于 AS. 芦苇栽种后于室外条件下缓苗 $10 \mathrm{~d}$. 每种底质条件下设置 9 个重复, 两种 底质共计 18 桶植物.

\section{2 实验方法}

缓苗结束后, 选取植株顶端第 3 片展开的叶片的中上部进行相对叶绿素含量和叶绿素菼光参数的测 定, 并以此作为淹水前 $(0 \mathrm{~d})$ 芦苇幼苗的相关指标. 然后将芦苇放人已注水的水池中, 水面位于芦苇植株顶 端 $20 \mathrm{~cm}$ 左右, 并根据实验期间芦苇的生长情况及时补水以保证淹水深度的稳定. 水下 $20 \mathrm{~cm}$ 处的光强约为 水面光强的 $23.6 \%$, 水池的水质状况见表 1 . 此后每 $5 \mathrm{~d}$ 取出每种底质上的 3 桶植物, 每片叶片的相对叶绿 素含量测定 5 次, 叶绿素荧光参数测定 2 次. 同时于淹水后的第 $10 、 20$ 和 $30 \mathrm{~d}$ 于每种底质上分别取出 3 桶 
同样进行相对叶绿素含量和叶绿素菼光参数的测定, 观察其淹水后的恢复状况, 并取芦苇上部的第 3 片叶 进行丙二醛含量 (MDA) 的测定. 实验共计 $40 \mathrm{~d}$. 恢复期间将水加至塑料桶上沿并及时补水以确保芦苇的恢 复过程中底质表层有 $5 \mathrm{~cm}$ 的水层.

相对叶绿素含量和叶绿素苂光均采用原位方法测定,前者采用便携式 SPAD-502 型叶绿素仪进行测定, 后者采用 DIVING-PAM(德国, WALZ 公司) 进行, 具体方法见参考文献 [10]. 叶片丙二醛含量的测定采用硫 代巴比妥酸法 (TBA 法) ${ }^{[11]}$.

表 1 实验中底质和水体的基本特征

Tab. 1 Primary properties of sediment and water in this experiment

\begin{tabular}{cccccc}
\hline 底质 & 湿容重 $/\left(\mathrm{g} / \mathrm{cm}^{3}\right)$ & 含水率 $/ \%$ & 烧失量 $/ \%$ & 总氮 $/(\mathrm{mg} / \mathrm{kg})$ & 总磷 $/(\mathrm{mg} / \mathrm{kg})$ \\
\hline $\mathrm{LS}$ & $1.524 \pm 0.324$ & $45.80 \pm 4.96$ & $7.56 \pm 0.82$ & $1451.5 \pm 97.3$ & $383.1 \pm 23.4$ \\
$\mathrm{AS}$ & $1.903 \pm 0.473$ & $28.97 \pm 3.47$ & $3.54 \pm 0.54$ & $453.4 \pm 67.2$ & $130.3 \pm 19.3$ \\
\hline \multirow{2}{*}{ 水体 } & $\mathrm{pH}$ & $\mathrm{COD}_{\mathrm{Mn}} /(\mathrm{mg} / \mathrm{L})$ & 氨氮 $/(\mathrm{mg} / \mathrm{L})$ & 总氮 $/(\mathrm{mg} / \mathrm{L})$ & 总磷 $/(\mathrm{mg} / \mathrm{L})$ \\
\cline { 2 - 7 } & $8.35 \pm 0.18$ & $10.23 \pm 1.95$ & $0.371 \pm 0.072$ & $1.048 \pm 0.103$ & $0.034 \pm 0.006$ \\
\hline
\end{tabular}

\section{3 数据处理}

采用 Excel 2003 和 SPSS 16.0 对数据进行分析处理和制图. 采用 One-way ANOVA 对数据间差异的显著 性进行检验.

\section{2 实验结果}

\section{1 淹水后芦苇叶片 rChlc 和 MDA 的变化}

缓苗过程中生长于 LS 上的芦苇其叶片 rChlc 较生长于 AS 的高 $11.17 \%$ 左右, 淹水后两种底质中的芦苇 叶片 rChlc 均下降, 且下降趋势相似 (图 1a). 到 $20 \mathrm{~d}$ 时, 生长于 LS 和 AS 上芦苇叶片的 rChlc 分别下降了 $13.78 \%$ 和 $18.17 \%$, 之后两者出现大幅下降, 至 $30 \mathrm{~d}$ 时分别比 $20 \mathrm{~d}$ 时下降了 $31.36 \%$ 和 $26.05 \%$. 方差分析 的结果表明, LS 和 AS 上芦苇叶片的 rChlc 均呈显著下降 $(P<0.01)$; 同时两种底质上芦苇 rChlc 的差异也达 到显著水平 $(P<0.01)$. 缓苗过程中 $\mathrm{MDA}$ 的变化也出现一定的差异, 生长于 $\mathrm{LS}$ 的芦苇叶片 $\mathrm{MDA}$ 较生长于 $\mathrm{AS}$ 的低 $17.96 \%$, 此后随着淹水时间的延长, 两种底质上芦苇叶片 MDA 均逐渐增加, 其中生长于 AS 上的芦 苇叶片 MDA 增速大于生长于 LS 上的, 两者的增加速率分别为 1.12 和 $0.68 \mathrm{nmol} /(\mathrm{g} \cdot \mathrm{d})$ (图 $1 \mathrm{~b}$ ). 方差分析 结果显示, 淹水 $30 \mathrm{~d}$ 时两种底质处理芦苇叶片 MDA 显著高于其它时间和底质处理 $(P<0.05)$ (其中 $20 \mathrm{~d}$ 时 AS 底质上的 MDA 已显著增加), 但两种底质间的差异不显著 $(P>0.05)$.
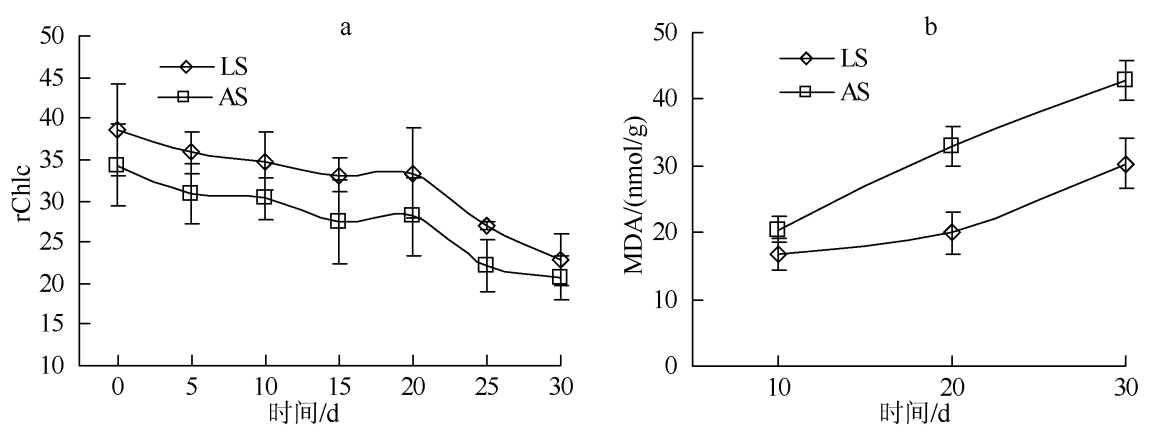

图 1 淹水期间两种底质上芦苇 $\operatorname{rChlc}(\mathrm{a})$ 和 $\operatorname{MDA}(\mathrm{b})$ 的变化

Fig. 1 Varieties of rChlc (a) and MDA (b) of P. communis in two types of sediment during submergence 


\section{2 淹水后芦苇叶片的叶绿素荧光特性的变化}

叶绿素菼光特性主要包括叶绿素苂光参数和快速光响应曲线两部分. 叶绿素苂光参数中本文采用 PS II 的最大量子产量 $F v / F m$ 和实际量子产量 $Y$ 两个指标. 缓苗过程中生长于 LS 中芦苇的 $F v / F m$ 和 $Y$ 分别比生 长于 AS 中的高 $2.34 \%$ 和 $18.35 \%$, 淹水后两种底质上芦苇的 $F v / F m$ 和 $Y$ 均呈下降趋势. $F v / F m$ 的下降速率 与 $Y$ 相比要慢, 淹水 $5 \mathrm{~d}$ 时 LS 和 $\mathrm{AS}$ 上芦苇的 $F v / F m$ 分别下降了 $3.43 \%$ 和 $10.58 \%$, 而 $Y$ 则分别下降了 $19.46 \%$ 和 $19.76 \%$; 生长于 LS 上芦苇的 $F v / F m$ 和 $Y$ 分别在淹水 $20 \mathrm{~d}$ 和 $15 \mathrm{~d}$ 后趋于稳定, 而生长于 $\mathrm{AS}$ 上的 则分别在淹水 $15 \mathrm{~d}$ 和 $5 \mathrm{~d}$ 后, 较 LS 有所提前, 但 AS 上的 $F v / F m$ 和 $Y$ 均小于 LS 上的(图 2). 方差分析表明, 两种底质上芦苇的 $F v / F m$ 和 $Y$ 均显著下降 $(P<0.05) ; F v / F m$ 在两种底质上的差异达到了显著水平 $(P<$ $0.05)$, 而 $Y$ 的差异则不显著 $(P>0.05)$. 另外, 将 $\mathrm{rChlc}$ 的变化与叶绿素苂光参数的变化进行相关性分析 (表 2), 结果表明淹水期间两种底质上芦苇叶片 rChlc 与叶绿素菼光参数间总体上呈显著正相关 $(P<$ $0.05)$ ，其中 AS 底质上 $F v / F m$ 与 rChlc 之间的相关性达到极显著水平 $(P<0.01)$.

表 2 相对叶绿素含量与叶绿素菼光参数的相关性分析 ${ }^{1)}$

Tab. 2 Correlation analysis of relative chlorophyll content and chlorophyll fluorescence parameters

\begin{tabular}{|c|c|c|c|c|c|c|c|c|}
\hline \multirow{2}{*}{ 底质 } & \multicolumn{2}{|c|}{ 淹水期间 } & \multicolumn{2}{|c|}{ A10 } & \multicolumn{2}{|c|}{$\mathrm{A} 20$} & \multicolumn{2}{|c|}{ A 30} \\
\hline & $F v / F m$ & $Y$ & $F v / F m$ & $Y$ & $F v / F m$ & $Y$ & $F v / F m$ & $Y$ \\
\hline LS & 0.800 * & 0.715 & $0.777^{*}$ & $0.958^{* *}$ & 0.357 & 0.103 & 0.927 & 0.901 \\
\hline AS & $0.880 * *$ & 0.854 * & $0.775^{*}$ & 0.712 & 0.661 & 0.844 & 0.968 & 0.949 \\
\hline
\end{tabular}

1) $\mathrm{A} 10 、 \mathrm{~A} 20$ 和 $\mathrm{A} 30$ 分别为淹水 $10 、 20$ 和 $30 \mathrm{~d}$ 后的恢复状况; 淹水期间和 $\mathrm{A} 10$ 中 $n=7, \mathrm{~A} 20$ 中 $n=5, \mathrm{~A} 30$ 中 $n=3$; 无标记 表示 $P>0.05, *$ 表示 $P<0.05, * *$ 表示 $P<0.01$.

快速光响应曲线 (RLCs) 是相对电子传递速率 $(r E T R)$ 随光强 $(P A R)$ 变化的连续曲线. 淹水后芦苇 RLCs 上的 $r E T R$ 即呈显著下降趋势, 最大 $r E T R$ 下降达 $50 \%$ 以上, 因此图 2 只给出了从淹水 $5 \mathrm{~d}$ 起 RLCs 的变化情 况. 随着淹水时间的延长,两种底质上芦苇的 rETR 逐渐下降,但是在下降方式上两者表现出一定的差异: 生 长于 LS 上的芦苇其下降过程分为两部分, 即淹水后 $25 \mathrm{~d}$ 内的缓慢下降过程和 $30 \mathrm{~d}$ 时的快速下降过程, $25 \mathrm{~d}$ 内最大 rETR 与 $5 \mathrm{~d}$ 时相比下降 28. 24\%,30 d 时下降达 58.71\%,25 30 d 的下降高达 $42.46 \%$; 而生长于 AS 上的则包括三个部分, 即淹水后 $15 \mathrm{~d}$ 内、 $20 \sim 25 \mathrm{~d}$ 内及 $30 \mathrm{~d}$ 时的快速下降过程, 淹水 $15 \mathrm{~d}$ 时较 $5 \mathrm{~d}$ 时仅 下降 5.62\% ,淹水 $20 、 25$ 和 $30 \mathrm{~d}$ 时分别下降了 $38.08 \%$ 、 $43.57 \%$ 和 $71.29 \%$ (图 2).

\section{3 淹水胁迫解除后芦苇的恢复状况}

2.3.1 芦苇叶片 rChlc 和 MDA 的变化 淹水 $10 、 20$ 和 $30 \mathrm{~d}$ 时 LS 底质上芦苇叶片的 rChlc 分别为 34.82 、 33.33 和 $22.88, \mathrm{MDA}$ 则分别为 $16.78 、 19.99$ 和 $30.38 \mathrm{nmol} / \mathrm{g}$; AS 底质上芦苇叶片的 rChlc 分别为 30.26 、 28.10 和 $20.78, \mathrm{MDA}$ 分别为 $20.46 、 32.96$ 和 $42.86 \mathrm{nmol} / \mathrm{g}$. 当淹水胁迫解除后, 芦苇叶片的 rChlc 均有所上 升, MDA 则逐渐下降( 图 3、4) : 淹水 $10 、 20$ 和 $30 \mathrm{~d}$ 后 LS 底质上的芦苇叶片 rChlc 较刚解除淹水胁迫后分别 增加 $9.99 \% 、 1.88 \%$ 和 $17.04 \%$, AS 底质上的则分别增加 $12.33 \% 、 15.50 \%$ 和 $18.24 \%$; 淹水 $10 、 20$ 和 $30 \mathrm{~d}$ 后 LS 底质上的芦苇叶片 MDA 分别下降 43. 46\%、40.68\% 和 $4.60 \%$, AS 底质上的分别下降 48. 24\%、 $37.81 \%$ 和 $9.34 \%$. 虽然 rChlc 的变化程度不同,但其大小仍然表现为 $10 \mathrm{~d}>20 \mathrm{~d}>30 \mathrm{~d}$; 同样, MDA 的大小 则表现为 $10 \mathrm{~d}<20 \mathrm{~d}<30 \mathrm{~d}$. 即淹水胁迫解除后芦苇叶片 rChlc 和 MDA 与淹水时间的长短有关, 淹水时间短 时芦苇叶片具有较高的 rChlc 和较低的 MDA, 反之亦然. 方差分析的结果表明, LS 上淹水 $30 \mathrm{~d}$ 后芦苇叶片的 MDA 显著高于其它时间处理 $(P<0.05)$, 淹水 $10 \mathrm{~d}$ 和 $20 \mathrm{~d}$ 后恢复生长, 芦苇叶片的 MDA 总体呈现出显著 下降 $(P<0.05)$, 之后下降趋势不显著; AS 上情况较为复杂, 但总体也表现出淹水 $30 \mathrm{~d}$ 后芦苇叶片的 MDA 较高, 淹水 $10 \mathrm{~d}$ 和 $20 \mathrm{~d}$ 后随着时间的延长总体呈现出显著的下降趋势 $(P<0.05)$. 同时, 两种底质间 $10 \mathrm{~d}$ 和 $20 \mathrm{~d}$ 的差异均达到了极显著水平 $(P<0.01), 30 \mathrm{~d}$ 的差异则不显著 $(P>0.05)$.

2.3.2 芦苇叶片的叶绿素荧光特性的变化 淹水 $10 、 20$ 和 $30 \mathrm{~d}$ 时 LS 底质上芦苇叶片的 $F v / F m$ 分别为 $0.724 、 0.656$ 和 $0.660, Y$ 分别为 $0.460 、 0.444$ 和 0.402 ; AS 底质上 $F v / F m$ 分别为 $0.643 、 0.612$ 和 $0.572, Y$ 分别为 $0.418 、 0.390$ 和 0.380 . 淹水胁迫解除后, 两者均呈增加趋势 (图 5). 淹水 $10 \mathrm{~d}$ 后 LS 底质上芦苇叶片 

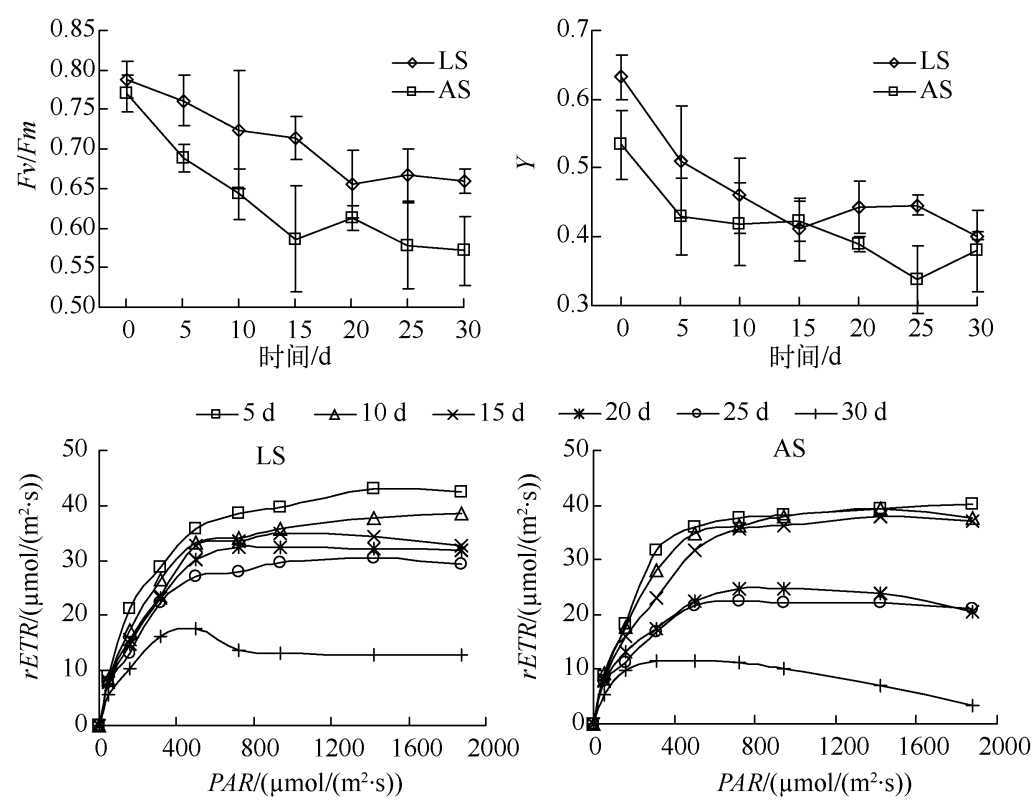

图 2 淹水期间叶绿素苂光特性

(PS II 的最大量子产量 $F v / F m$, 实际量子产量 $Y$ 和快速光响应曲线) 的变化

Fig. 2 Varieties of chlorophyll fluorescence characteristics(the maximal quantum yield of PS II $(F v / F m)$, actual quantum yield $(Y)$ and rapid light curves $)$ during submergence
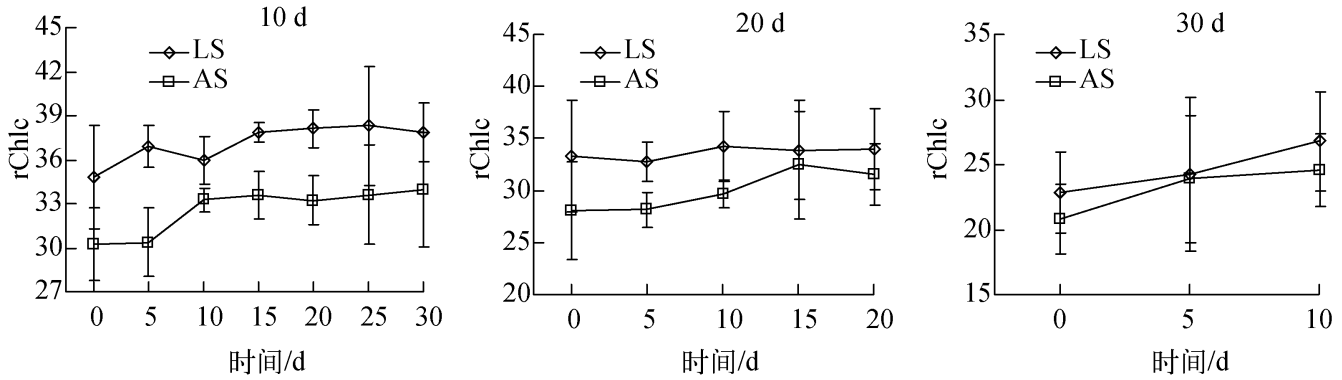

图 3 恢复期间不同淹水时间后芦苇叶片 rChlc 的变化

Fig. 3 Varieties of rChlc after different extension of submergence in recovery period

的 $F v / F m$ 和 $Y$ 分别增加 $8.96 \%$ 和 $28.28 \%, A S$ 底质上则分别增加 $22.40 \%$ 和 $40.29 \%$; 淹水 $20 \mathrm{~d}$ 后 LS 底质 上 $F v / F m$ 和 $Y$ 分别增加 $16.34 \% 、 30.51 \%, \mathrm{AS}$ 底质上分别增加 $19.77 \% 、 47.88 \%$. 淹水 $30 \mathrm{~d}$ 后 LS 底质上 $F v /$ $F m 、 Y$ 分别增加 $6.52 \% 、 24.78 \%$, AS 底质上分别增加 $10.51 \% 、 25.85 \%$. 两种底质上芦苇叶片的 $F v / F m$ 和 $Y$ 均表现为 $10 \mathrm{~d}>20 \mathrm{~d}>30 \mathrm{~d}$, 即淹水胁迫解除后芦苇叶片的 $F v / F m$ 和 $Y$ 与淹水时间的长短有关, 淹水时间短 时芦苇叶片则具有较高的 $F v / F m$ 和 $Y$. 淹水 $10 \mathrm{~d}$ 和 $20 \mathrm{~d}$ 后芦苇叶片的 $F v / F m$ 和 $Y$ 均显著增加 $(P<0.05)$, 淹水 $30 \mathrm{~d}$ 后增加不显著 $(P>0.05)$; 两种底质上芦苇的 $F v / F m$ 和 $Y$ 的差异也没有达到显著水平 $(P>$ 0.05 ). 同时淹水胁迫解除后随着时间的延长 RLCs 也呈逐渐增加的趋势 (图 6), 最大 $r E T R$ 分别由解除淹水 胁迫时 LS 底质上的 $38.50 、 32.45$ 和 $16.15 \mu \mathrm{mol} /\left(\mathrm{m}^{2} \cdot \mathrm{s}\right)$ 增加到 $125.10 、 92.00$ 和 $29.10 \mu \mathrm{mol} /\left(\mathrm{m}^{2} \cdot \mathrm{s}\right)$; AS 底质上的最大 $r E T R$ 分别由 39.25、24.80 和 $11.50 \mu \mathrm{mol} /\left(\mathrm{m}^{2} \cdot \mathrm{s}\right)$ 增加到 108.60、78.70 和 20.00 $\mu \mathrm{mol} /\left(\mathrm{m}^{2} \cdot \mathrm{s}\right)$, 即 $r E T R$ 的大小同样表现为 $10 \mathrm{~d}>20 \mathrm{~d}>30 \mathrm{~d}$. 淹水胁迫解除后两种底质上芦苇叶片 rChlc 和叶绿素荧光参数的相关性分析表明 (表 2), 淹水 $10 \mathrm{~d}$ 后芦苇叶片 $\mathrm{rChlc}$ 和叶绿素苂光参数之间总体上呈 显著正相关 $(P<0.05)$, 其中 $\mathrm{LS}$ 底质上 rChlc 与 $Y$ 的相关性达到极显著水平 $(P<0.01)$. 

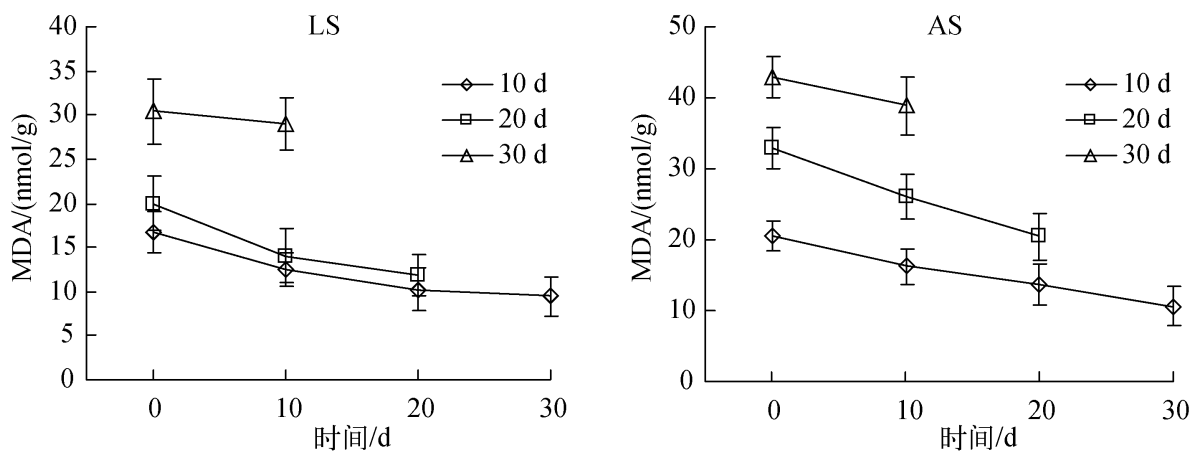

图 4 不同淹水时间后芦苇叶片 MDA 的变化

Fig. 4 Varieties of MDA of $P$. communis after different extension of submergence in recovery period
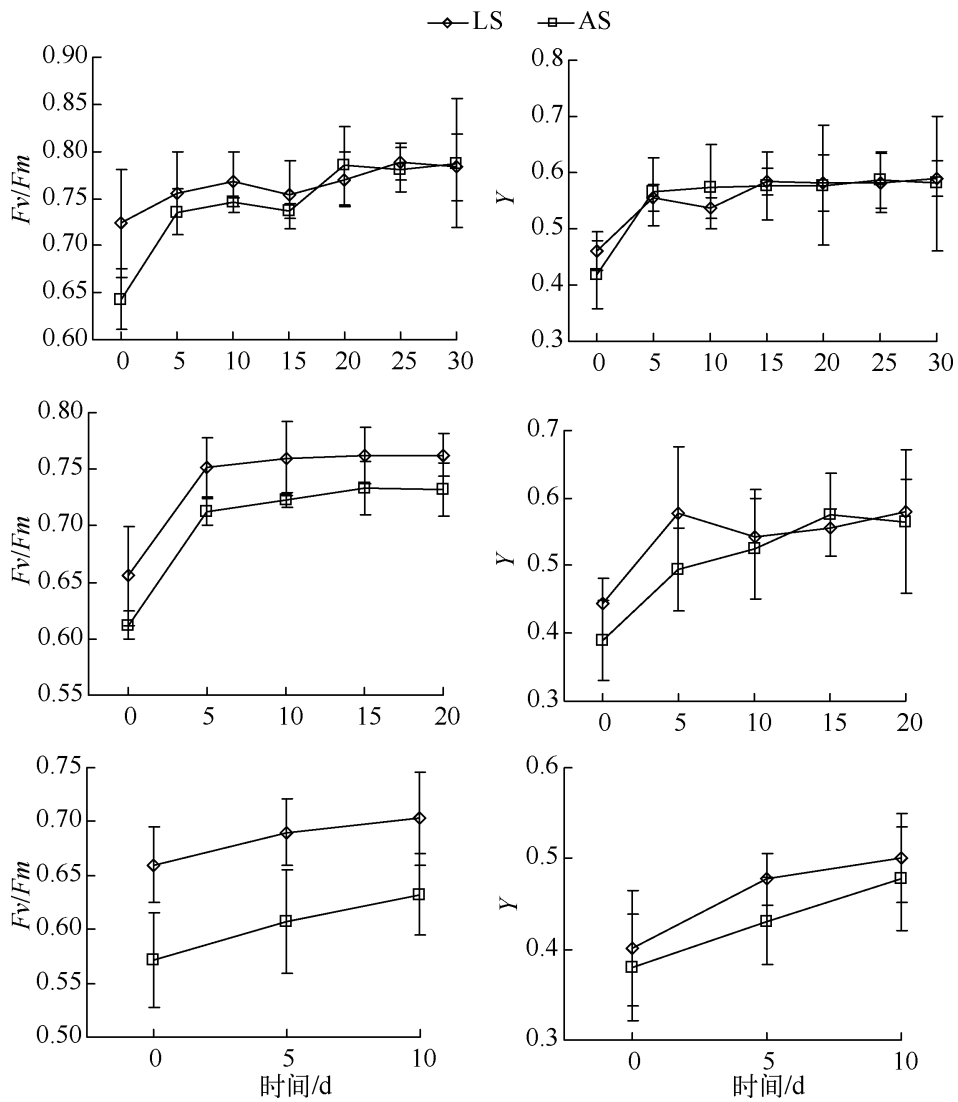

图 5 不同淹水时间后叶绿素苂光参数的变化

Fig. 5 Varieties of chlorophyll fluorescence parameters after different extension of submergence

\section{3 讨论}

\section{1 湿地植物对淹水的响应}

淹水环境不同于陆地环境, 这主要表现在水中较低的 $\mathrm{O}_{2}$ 和 $\mathrm{CO}_{2}$ 扩散速率及不断积累的乙烯含量, 还有 较低的光照强度等, 这种低氧弱光的淹水环境能够抑制植物光合作用的进行 ${ }^{[3,12-13]}$, 主要方式有: 淹水引起 

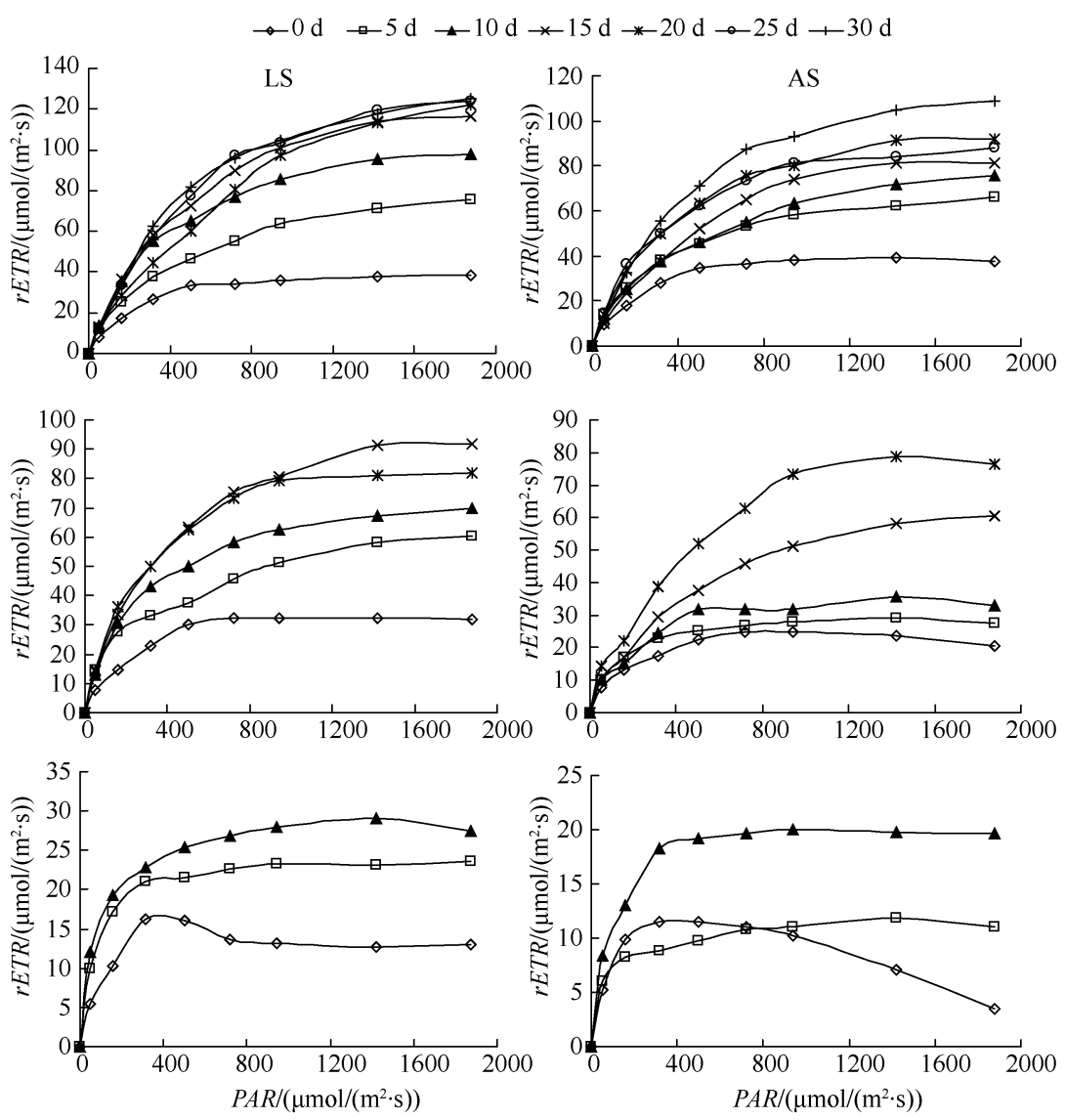

图 6 不同淹水时间后 RLCs 的变化

Fig. 6 Varieties of RLCs after different extension of submergence

湿地植物气孔关闭, 限制气体扩散 ${ }^{[14]}$; 降低植物的叶水势, 破坏叶绿素结构, 降低参与光合作用的酶活性和 光合产物的运输能力 ${ }^{[15]}$; 水中 $\mathrm{CO}_{2}$ 含量过低和叶片表皮限制 $\mathrm{CO}_{2}$ 进人从而抑制水下光合作用的进行 ${ }^{[16-17]}$; 湿地土壤氧化还原电位降低产生植物性毒素抑制植物的光合作用 ${ }^{[18]}$. 而大量的研究也表明, 光合系统中的 PS II 对各种逆境环境的胁迫非常敏感 ${ }^{[19-21]}$, 有关淹水对 PS II 功能影响的研究也有所报道 ${ }^{[22-23]}$. 叶绿素菼光 技术以光合作用理论为基础, 而光合作用受到伤害的最初部位与 PS II 紧密相连 ${ }^{[24]}$, 因此利用叶绿素苂光技 术来监测逆境对植物的影响在实际中得到了广泛应用. 叶绿素苂光参数中, $F v / F m$ 和 $Y$ 分别反映了 PS II 反 应中心内禀光能转换效率或称最大 PS II 的光能转换效率和 PS II 反应中心在有部分关闭情况下的实际原初 光能捕获效率, 后者是植物实际光合速率的表征. 本研究结果表明, 淹水对芦苇叶片的 PS II 造成一定的影 响, $F v / F m$ 和 $Y$ 均随着淹水时间的延长而逐渐下降. 相关研究也表明, $F v / F m$ 不受植物物种的影响, 在非胁 迫条件下该参数的变化极小, 但当植物受到环境条件的胁迫时则会显著下降 ${ }^{[25]}$. 一般高等植物的 $F v / F m$ 介 于 $0.70 \sim 0.85$ 之间, 多数为 $0.83^{[26]}$, 低于此值说明植物受到逆境环境的胁迫. 在本研究中, 淹水 $5 \mathrm{~d}$ 时, 虽 然两种底质上的芦苇叶片 $F v / F m$ 有所下降, 但生长于 AS 上的已降至 0.70 以下, 而生长于 LS 上的则到 $15 \mathrm{~d}$ 后才降至 0.70 以下, 因此生长于 AS 上的芦苇对淹水胁迫更为敏感; $Y$ 则具有相同的变化趋势, 即淹水后两 种底质上芦苇叶片的 $Y$ 均显著下降, 表明淹水降低了芦苇的光合速率. 快速光响应曲线 (RLCs) 是了解植物 光合作用对光强响应的重要手段, 淹水后随着淹水时间的延长, 两种底质上芦苇叶片的最大 $r E T R$ 也逐渐下 降, 与 $F v / F m$ 的变化趋势相同,生长于 $\mathrm{LS}$ 上的芦苇起初最大 $r E T R$ 下降较缓, $25 \mathrm{~d}$ 后则明显下降, 而生长于 AS 上的则在 $15 \mathrm{~d}$ 后即明显下降, 且 $25 \mathrm{~d}$ 后又有一个显著的下降过程. 可见,底质的营养状况对于芦苇在抵 
抗淹水胁迫及淹水胁迫解除后的光合作用恢复方面具有重要影响, 相对于 AS, 营养状况良好的 LS 能够使芦 苇具有较强的抗淹水能力, 相应也具有较强的光合作用能力. 本研究是在植株完全淹水的条件下进行的, 植 物的光合能力呈下降趋势. 但 $\mathrm{Li}$ 等的研究表明 ${ }^{[27]}$, 在芦苇植株不受淹的情况下, 其生长的土壤受淹与否, 植 株的净光合速率无明显差异, 这一方面可能与淹水后水下的光照强度显著下降导致芦苇光合能力下降有 关;另一方面芦苇具有发达的根状茎,只有在土壤淹水的条件下能够保证地下部分的正常生命活动.

\section{2 淹水后湿地植物的恢复生长}

当植物由水生环境暴露到空气中时, 就由弱光低氧环境转为强光高氧环境. Ella 等对水稻苗出水后的研 究表明, 叶片的光合机构在强光下可能会产生较高水平的活性氧 (AOS $)^{[28]}$, 从而使植物受到较大的氧化伤 害, 导致光合机构的光抑制, 使植物光合作用受阻,严重者将导致植物死亡. 淹水胁迫解除后, 植株的存活与 否很大程度上与植株在淹水条件下的营养储备和能量转化有关. 淹水条件下植物的光合作用受到抑制, 植 物为维持其生存必然要消耗自身已储备的有机物, 这往往造成淹水后植株的营养储备不足, 其基础代谢不 能得到保证, 甚至可导致植株死亡 ${ }^{[29-30]}$. 随着淹水时间的延长, 植株体内的营养消耗加剧, 营养储备降低, 从 而影响植株在淹水后的存活率 ${ }^{[31-32]}$. 相关研究表明, 植株在淹水结束后的恢复生长能力与该植株在淹水环 境中的生物量变化无显著相关性,一些能够很好地进行恢复生长的物种在淹水条件下的生物量呈不同的变 化趋势, 这可能与植株在两种不同环境下其生物量变化有着不同的生理学基础有关,并且一些耐淹物种的 恢复能力与其淹水时间的长短也没有直接的联系 ${ }^{[33]}$. 本研究中, 随着淹水时间的延长, 两种底质上芦苇植株 逐渐变得瘦弱,并导致生物量的下降. 实验结束时,淹水 $30 \mathrm{~d}$ 的两种底质上的芦苇叶片逐渐调落殆尽,退水 后植株茎节部和基部萌发出新叶和新的植株, 因此长时间的淹水能够削弱芦苇的生长, 但退水后则能够通 过萌发出新叶及新株来快速地适应环境. 同时两种底质上芦苇在淹水 $10 \mathrm{~d}$ 后其叶片的 rChlc 和叶绿素苂光 参数间总体存在显著正相关, 表明叶片的叶绿素含量还是其光合作用的主要限制因素, 这进一步表明叶片 的光合机构没有受到破坏. 但淹水 $20 \mathrm{~d}$ 后 rChlc 和叶绿素苂光参数没有显著相关性,表明芦苇叶片的光合机 构可能在一定程度上受到淹水的影响, 叶片叶绿素含量的增加不能引起光合能力的显著提升.

可见,淹水环境抑制了幼苗期芦苇的生长, 表现在叶片中叶绿素含量的减少、膜脂过氧化作用的增加, 最大 PS II 的光能转换效率的降低以及实际光合速率的下降,且叶绿素含量的减少与菼光参数的下降间总体 呈现出显著的正相关关系, 同时光合作用过程中的电子传递速率及对强光的耐受能力也有所下降; 对生长 于 AS 上芦苇的抑制程度要大于生长于 LS 上的,这在一定程度上表明提高底质的营养状况可以增加芦苇抗 淹水的能力. 在淹水 $20 \mathrm{~d}$ 内解除胁迫, 芦苇可以通过叶片光合功能的恢复来恢复生长, 而淹水达 $30 \mathrm{~d}$ 时, 芦 苇的生长则需要通过新叶和新的植物体来维持. 因此, 芦苇具有一定的抗淹水能力, 但是淹水将延缓芦苇种 群的形成, 从而延缓湖滨湿地的恢复进度. 然而, 本研究仅从生理方面对淹水环境中芦苇幼苗对两种底质的 响应及淹水胁迫解除后的恢复情况进行了研究,但是关于淹水环境中芦苇幼苗对底质的响应和淹水胁迫解 除后的恢复状况的形态等其它指标, 以及成熟植株对底质的响应、芦苇种群可以通过个体间的互惠作用等 适应淹水环境等方面的研究还有待于进一步加强.

\section{4 参考文献}

[ 1 ] 王海洋,陈家宽, 周 进. 水位梯度对湿地植物生长、繁殖和生物量分配的影响. 植物生态学报, 1999,23 (3): 269-274.

[2] 徐治国,何 岩,间百兴等. 营养物及水位变化对湿地植物的影响. 生态学杂志,2006,25(1):87-92.

[ 3 ] 刘云峰,秦洪文,石 雷等. 水淹对水芹叶片结构和光系统 II 光抑制的影响. 植物学报, 2010,45(4):426-434.

[ 4 ] 李文朝. 五里湖底质条件与水生高等植物的适应性研究. 湖泊科学, 1996,8(增刊) :30-36.

[ 5 ] Brix H. Functions of macrophytes in constructed wetlands. Water Science and Technology, 1994, 29(4) : 71-78.

[6]方云英,杨肖娥,常会庆等. 利用水生植物原位修复污染水体. 应用生态学报, 2008,19(2):407-412.

[ 7 ] 谢 平. 翻阅巢湖的历史一一蓝藻、富营养化及地质演化. 北京:科学出版社,2009.

[8] 阎伍玖,王心源. 巢湖流域非点源污染初步研究. 地理科学, 1998,18 (3) :263-267.

[9] 殷福才,张之源. 巢湖富营养化研究进展. 湖泊科学,2003,15(4):377-384.

[10］柏 祥,陈开宁,黄 蔚等. 黄菖蒲和美人蕉对水深梯度的响应差异. 生态学杂志,2011,30(3):464-470. 
[11］常福辰,陆长梅, 沙 莎. 植物生物学实验. 南京: 南京师范大学出版社, 2007 .

[12] Franklin P, Dunbar M, Whitehead P. Flow controls on lowland river macrophytes: A review. Science of the Total Environment, 2008, 400: 369-378.

[13] Sand-Jensen K. Environmental variables and their effect on photosynthesis of aquatic plant communities. Aquatic Botany, $1989, \mathbf{3 4}(1 / 2 / 3): 5-15$.

[14] 范晓荣,沈其荣. ABA、IAA 对旱作水稻叶片气孔的调节作用. 中国农业科学, 2003,36(2) : 1450-1455.

[15] Pezeshki SR, Anderson PA. Responses of three bottom land woody species with different flood-tolerance capabilities to various flooding regimes. Wetland Ecology and Management, 1997, 4: 245-256.

[16] Mommer L, de Kroon H, Pierik R et al. A functional comparison of acclimation to shade and submergence in two terrestrial plant species. New Phytologist, 2005, 167: 197-206.

[17] 陈鹭真,林 鹏,王文卿. 红树植物淹水胁迫响应研究进展. 生态学报,2006,26(2):586-593.

[18］卢 妍. 湿地植物对淹水条件的响应机制. 自然灾害学报,2010,19(4):147-151.

[19] Jiang CD, Jiang GM, Wang XZ et al. Increased photosynthetic activities and thermostability of photosystem II with leaf development of elm seedlings (Ulmus pumila) probed by the fast fluorescence rise OJIP. Environmental and Experimental Botany, 2006, 58(1/2/3) : 261-268.

[20］赵 昕,吴雨霞,赵敏桂等. $\mathrm{NaCl}$ 胁迫对盐芥和拟南芥光合作用的影响. 植物学通报,2007,24:154-160.

[21］李洪燕,郑青松, 刘兆普等. 海水胁迫对苦荬菜幼苗生长及生理特性的影响. 植物学报, 2010,45(1):73-78.

[22] Fernández MD. Changes in photosynthesis and fluorescence in response to flooding in emerged and submerged leaves of Pouteria orinocoensis. Photosynthetica, 2006, 44(1) : 32-38.

[23] 衣英华,紮大勇,谢宗强等. 模拟淹水对枫杨和栓皮栋气体交换、叶绿素菼光和水势的影响. 植物生态学报,2006, 30 : $960-968$.

[24] Havaux M, Florence T. Temperature-dependent adjustment of thermal stability of photosystem II in vivo: possible involvement of xanthophylls-cycle pigments. Planta, 1996, 198: 324-333.

[25] 韩博平, 韩志国, 付 翔. 藻类光合作用机理与模型. 北京:科学出版社, 2003 .

[26] Demmig B, Björkman O. Comparison of the effect of excessive light on chlorophyll fluorescence (77K) and photon yield of $\mathrm{O}_{2}$ evolution in leaves of higher plants. Planta, 1987, 171: 171-184.

[27] Li M, Yang D, Li W. Leaf gas exchange characteristics and chlorophyll fluorescence of three wetland plants in response to long-term soil flooding. Photosynthetica, 2007, 45(2) : 222-228.

[28 ] Ella ES, Kawano N, Ito O. Importance of active oxygen-scavenging system in the recovery of rice seedlings after submergence. Plant Science, 2003, 165: 85-93.

[29] 王海锋, 曾 波, 李 娅等. 长期完全水淹对 4 种三峡库区岸生植物存活及恢复生长的影响. 植物生态学报, 2008, 32 ( 5 ) : $977-984$.

[30 ] Rawyler A, Arpagaus S, Braendle R. Impact of oxygen stress and energy availability on membrane stability of plant cells. Annals of Botany, 2002, 90 : 499-507.

[31] Vartapetian BB, Jackson MB. Plant adaptations to anaerobic stress. Annals of Botany, 1997, 79 (Suppl. A) : 3-20.

[32] Crawford RMM, Braendle R. Oxygen deprivation stress in a changing environment. Journal of Experimental Botany, 1996, 47(2): 145-159.

[33] van Eck WHJM, van de Steeg HM, Blom CWPM et al. Is tolerance to summer flooding correlated with distribution patterns in river floodplains? A comparative study of 20 terrestrial grassland species. OIKOS, 2004, 107: 393-405. 